\title{
Vasa previa: Prenatal diagnosis and management
}

Yaakov MELCER ${ }^{1}$, MD; Ron MAYMON'1, M.D. and Eric JAUNIAUX², MD, PhD

${ }^{1}$ Department of Obstetrics and Gynecology, The Yitzhak Shamir Medical Center (formerly Assaf Harofeh Medical Center), Zerifin, Israel, affiliated with the

Sackler School of Medicine, Tel-Aviv University, Tel-Aviv, Israel; ${ }^{2}$ Academic

Department of Obstetrics and Gynaecology, EGA Institute for Women's Health,

Faculty of Population Health Sciences, University College London (UCL),

London, UK

No funding was obtained for this study.

The authors report no conflict of interest.

"Corresponding author: Professor Eric Jauniaux, Academic Department of Obstetrics and Gynaecology, Institute for Women's Health, University College London, 86-96 Chenies Mews, London WC1E 6HX, UK. Telephone numbers: $+44 / 207 / 3908113$ Fax: $+44 / 207 / 3908115$

E-mail: e.jauniaux@ucl.ac.uk

Keywords: vasa previa, prenatal diagnosis, management, ultrasound, cervical length

Word count main text: 2047 
ABSTRACT (200 words)

Purpose of review: Vasa previa (VP) is a rare disorder of placentation associated with a high rate of perinatal morbidity and mortality when undetected before delivery. We have evaluated the recent evidence for prenatal diagnosis and management of VP.

Recent findings: Around $85 \%$ of cases of VP have one or more identifiable risk factors including in vitro fertilization (IVF), multiple gestations, bilobed, succenturiate or low-lying placentas and velamentous cord insertion. The development of standardized prenatal targeted scanning protocols may improve perinatal outcomes. There is no clear consensus on the optimal surveillance strategy including the need for hospitalization, timing of corticosteroids administration and the value of transvaginal cervical length measurements. Outpatient management is possible if there is no evidence of cervical shortening on ultrasound and there are no symptoms of bleeding or uterine contractions. Recent national guidelines and expert reviews have recommended scheduled cesarean section of all asymptomatic women presenting with VP between 34 and 36 weeks' gestation.

Summary: Prenatal diagnosis of VP is pivotal to prevent intrapartum fetal death. Although there is insufficient evidence to support the universal midgestation ultrasound screening for VP, recent evidence indicates the need for standardized prenatal targeted screening protocols of pregnancies at high-risk of VP. 


\section{INTRODUCTION}

Vasa previa (VP) results from an anomaly of placentation which is defined as the presence of an aberrant fetal vessels running through the membranes across the internal os of the cervix under the fetal presentation [1-3]. There are 2 types of VP depending if the free vessel is connected to a velamentous cord (type I) or connected to a succenturiate or accessory lobe of the main placenta with (type II).

The VP is unprotected by the Wharton jelly of the umbilical cord or the tissue of the placental chorionic plate and thus exposed to rupture during the spontaneous rupture of the membranes of an active labour or at amniotomy to induce or augment early labour. The classic presentation of undiagnosed VP during labour is the presence of painless vaginal fresh bleeding. Because the total fetal blood circulating volume at term is around 80 to $100 \mathrm{~mL} / \mathrm{kg}$, the loss of what may appear to be a relatively small amount of blood can have major impact on the fetus and be rapidly fatal [4-6].

The incidence of VP has been reported to range between in 1 in 500 to 1 in 5000 pregnancies [1-6]. Similarly, to other disorders of placentation, i.e. placenta previa and placenta accreta spectrum, there has been mounting clinical evidence over the last decade that prenatal diagnosis of VP can have a direct impact on perinatal outcome $\left[6-8,9^{*}, 10^{\star \star}\right]$. Although it has been estimated that around 150 fetuses die at birth due to VP in the UK alone [11], there is still a lot of controversy regarding universal screening of VP, mainly due limited prospective data available $[12,13]$. Recently, several epidemiological studies, expert reviews and systematic reviews and meta-analysis have indicated that 
pregnancies resulting from assisted reproduction technology (ART), presenting with a low-lying placenta, a velamentous umbilical cord insertion and multiple pregnancies are at higher risk of VP $\left[14-17,18^{\star *}, 19^{\star *}\right]$. These findings suggests that targeted screening for VP might be cost-effective $\left[10^{\star \star}\right]$. The present review presents the latest evidence on the epidemiology, prenatal diagnostic techniques and screening strategies and management of VP.

\section{REVIEW}

\section{Epidemiology}

A recent systematic review of 13 studies (two prospective cohort studies, 10 retrospective cohort studies and one case-control study) and reporting on 569410 women found a mean incidence of 0.60 per 100 pregnancies. The authors found that $83 \%$ of the 325 VP cases reviewed had one or more risk factor $\left[18^{* *}\right]$. The highest Odd Ratio (OR) was found for the presence of a velamentous cord insertion (VCI) (OR 672;95\% Confidence Intervals (CI) 1124034) followed by placental morphological anomalies i.e. bilobed placenta and succenturiate placental lobes, conception by ART; second trimester low-lying placenta and multiple gestations (Table 1). These data were confirmed by the recent prospective population-based cohort study undertaken using the Australasian Maternity Outcomes Surveillance System and showing that 55 (95\%) of the 58 women had at least one risk factor for VP with VCI (62\%) and low-lying placenta (60\%) the most prevalent $\left[19^{* *}\right]$.

Pregnancies conceived by ART and in particular in vitro fertilization (IVF) have consistently been associated with a higher incidence of abnormal cord 
insertion $\left[20^{* *}, 21-25\right]$. The incidence of VP has been reported to be as high as one in 200 pregnancies conceived after ART and is likely to increase in the next decade with the increase use of ART worldwide $\left[10^{* *}\right]$. It has been hypothesized that VP could be due to the inadequate orientation of the IVF blastocyst during implantation or to a higher incidence of vanishing twins in IVF than in spontaneous twins $\left[16,20^{\star *}\right]$.

Our recent case series and systematic review has indicated a total incidence of $11.0 \%$ of VP in twin pregnancies [20**]. Like for singleton pregnancies, a $\mathrm{VCl}$ was the most common additional ultrasound findings in twins presenting with VP. The incidence of succenturiate lobes and other morphological placental anomalies is higher in twin pregnancies $[1,17,24,26]$. A $\mathrm{VCl}$ of one of the umbilical cords is eight times more common in twins than in singletons [26]. The OR for vasa previa in pregnancies presenting with a bilobate placenta or with succenturiate lobe has been evaluated to be 22.1 in twin pregnancies [26]. Five of the seven cases in our series and five out of the eight cases described in details in the literature presented with a $\mathrm{VCI}\left[20^{* \star}\right]$. One cases in our series and three in the literature also presented with a lowlying placenta.

\section{Prenatal diagnosis}

Gianopoulos et al. were the first to report in 1987 a case of VP diagnosed by ultrasound at 25 weeks of gestation in a pregnancy presenting with a low-lying placenta and a $\mathrm{VCl}$ on a succenturiate lobe [27]. Multiple case reports and small retrospective cohort series were published over the last three decades. The largest study published so far included 155 women with VP confirmed at 
delivery [6]. This study was the first to highlight the impact of prenatal diagnosis of VP on perinatal outcome with a $97 \%$ survival rate in cases of diagnosed antenatally compared with only $44 \%$ when the diagnosis was made during delivery. Recent studies have shown that prenatal diagnosis in centres with prenatal diagnosis expertise can be associated with $100 \%$ neonatal survival $\left[10^{* *}, 19^{\star *}\right]$

VP was first diagnosed using gray-scale ultrasound imaging as parallel or circular echogenic lines near the internal cervical os [27]. The advent of colour Doppler imaging (CDI) has allowed a more precise diagnosis by demonstrating that these lines to be vessels with pulsed Doppler showing a fetal arterial or venous waveform (Figure 1A-D). The combined used gray-scale and CDI is helpful in the differential diagnosis between VP from placental marginal sinus (where pulsed Doppler reveals maternal vessel waveform) and from umbilical cord presentation (easily displaced by gentle pressure on the maternal abdomen) [7]. New development in ultrasound imaging such as fourdimensional spatiotemporal image correlation (4D-STIC) may improve the diagnostic accuracy of VP [28].

A systematic review of the diagnostic accuracy of ultrasound imaging in the diagnosis of VP including two prospective and six prospective studies has found that the prenatal detection rates during second trimester ranging between $53 \%$ to $100 \%$ [29]. Four of the studies used transvaginal ultrasound (TVS) as the primary method for VP diagnosis whereas the remaining four used transabdominal ultrasound followed by TVS for confirmation of diagnosis. The only two prospective studies were using TVS CDI and have demonstrated high 
diagnostic rates with sensitivities of $100 \%$ and sensitivity of $99.0-99.8 \%[30,31]$ Hasegawa et al. have shown that pregnancies at risk of VP could be identified as early as 9 weeks of gestation [32]. However, as the definitive placenta is only fully formed by 10-11 weeks of gestation making it impossible in many cases to predict the final position of the umbilical cord and to identify placental anomalies such as bilobed placenta, there appears to be little benefit in attempting to identify VP during the first trimester. Overall, the prenatal diagnosis remains more effective around mid-gestation (18-26 weeks) than during the first or third trimester.

Universal screening for VP is an attractive option to reduce perinatal mortality, but evidence in support of this strategy is unsupported by evidence. The incidence of VP in the general population is lower than most fetal anomalies, its prevalence difficult to evaluate as not all placenta are examined at birth for this type of anomaly and the sensitivity of ultrasound of accurately screening for it difficult to assess as studies have been conducted in specialist centres and are mainly retrospective. A recent decision and cost-effectiveness analysis comparing four screening strategies for the prenatal screening of VP in singleton pregnancies has shown that that screening pregnancies conceived by IVF is the most cost-effective strategy with an incremental cost effectiveness ratio of $\$ 29,186.50$ / quality adjusted life years $\left[33^{* *}\right.$.

Identification of the placental cord insertion during at the mid-gestation ultrasound examination to exclude a $\mathrm{VCl}$ which is the main risk factor for VP, is easy and accurate, takes less than 1 minute and requires no additional scanning skills for a trained sonographer (Figure 3). However, there is no 
recommendation for routine screening for VP as part of the complete ultrasound examination, and ultrasound diagnosis of VP is not routinely taught during ultrasound training courses. We have recently shown that the implementation of standardized prenatal targeted scanning protocols for pregnant women with risk factors for VP improves the prenatal diagnosis and thus the perinatal outcome. Our study included 51 cases with confirmed VP at delivery and the prenatal detection rate increased after implementation of the screening protocol (9 of 18 cases [50\%] vs 29 of 33 cases [87.9\%]. Accurate prenatal diagnosis was shown to reduce the risks of perinatal complications mainly low Apgar scores and improves umbilical cord $\mathrm{pH}$ and haemoglobin levels for newborns with vasa previa $\left[10^{\star *}\right]$.

\section{Management}

There is currently no clear consensus on the optimal surveillance strategy including the need for antepartum hospitalization, timing of corticosteroids administration and the value of transvaginal cervical length measurements. There are so far no randomised control trails (RCTs) to support any of the recommendation presented below. Overall management strategies are empirical and based on not being able to predict the optimal timing of delivery for individual women.

Recent national guidelines and expert reviews have recommended scheduled cesarean section of all asymptomatic women presenting with vasa previa between 34 and 36 weeks' gestation $\left[33^{* \star}, 34,35,36^{\star}, 37\right]$. A decision analysis comparing 11 strategies for delivery timing in a patient with VP found that scheduled delivery at 34 weeks of gestation was the preferred strategy and 
resulted in the highest quality-adjusted life-years under the base-case assumptions [38]. The authors also found no benefit to expectant management beyond 36 weeks of gestation and amniocentesis for verification of fetal lung maturity does not improve outcomes.

There is also is weak evidence based on low-quality data supporting systematic antenatal hospitalisation in a unit with neonatal facilities from 30-32 weeks of gestation $\left[33^{* *}\right]$. The purpose of hospitalization is to allow for closer surveillance for signs of labour, corticosteroids administration to enhance fetal lung maturation and a timelier performance of cesarean delivery before labour and/or membrane rupture. The data of the recent prospective population-based cohort study using the Australasian Maternity Outcomes Surveillance System found no difference in perinatal outcome when VP was diagnosed prenatally between women who were hospitalised compared to those with no antenatal hospitalisation $\left[19^{\star \star}\right]$.

Sonographic measurements of cervical length $(\mathrm{CL})$ have been shown to be useful in predicting and managing women at risk for preterm delivery [39], as well as predicting the risk of emergent cesarean delivery (CD) in placenta previa $[40,41]$. The role of TVS CL measurements in the management of pregnancies with VP has only been evaluated recently. In a retrospective case-control study of 29 singleton pregnancies diagnosed with of VP on ultrasound in the second, we found that the rate of $\mathrm{CL}$ shortening is significantly slower for women with elective CD compared with those needing an emergent caesarean section [42*]. For each additional millimetre-per-week decrease in CL, the odds of emergency CD were increased by $6.50(95 \% \mathrm{Cl} 1.02-41.20)$. Our data suggest that serial 
cervical length measurements should start from $26-28$ weeks. If the cervix is shorter or shortening more rapidly, the patient should be hospitalized and administered corticosteroids to enhance fetal lung maturation. The CD could be planned by section at 34-36 weeks if the patient remains asymptomatic (Figure 2).

Like singletons, twin pregnancies presenting with VP have very poor perinatal outcomes if the diagnosis is not made before the onset of labor. Twin pregnancies are at higher risks of preterm delivery and a recent retrospective cohort study of 441 women with twin pregnancies has found that integration of serial measurements of CL using a stepwise algorithm in asymptomatic women with twin gestations can improve the detection of women at risk of preterm birth [43]. To minimize the impact of prematurity, twins diagnosed with VP with uncomplicated antenatal course and stable CL, could be scheduled for caesarean delivery at 36 weeks. However, the data on CL from our series and from the literature suggest that a $C L \geq 30 \mathrm{~mm}$ could be a better limit to allow for conservative management in women with twins and vasa previa. The role of $\mathrm{CL}$ measurements in the management of singleton $s$ and twins presenting with VP need to be prospectively evaluated by a multicentric study.

\section{CONCLUSION (word count 99)}

Prenatal diagnosis of VP prevents fetal demise during delivery in both singleton and twin pregnancies. Although there is insufficient evidence to support a universal mid-gestation ultrasound screening for VP, recent epidemiological data support the development of standardized ultrasound screening protocols 
targeting pregnancies at high-risk of VP i.e. presenting with VCI, conceived with IVF, multiple pregnancy, presenting with low-lying placenta or placental shape anomalies. Serial measurements of CL in pregnancies diagnosed with VP may play a role in the management pathways of both singletons and twins and provide individualized care in particular regarding hospitalization and timing corticosteroids administration and delivery.

\section{Key points:}

- Prenatal diagnosis of VP is essential to prevent fetal death.

- There is no clear consensus on the optimal surveillance strategy including the need for hospitalization, timing of corticosteroids administration and the value of transvaginal cervical length measurements.

- Outpatient management is possible if there is no evidence of cervical shortening on ultrasound and there are no symptoms of bleeding or uterine contractions.

- Recent national guidelines and expert reviews have recommended scheduled cesarean section of all asymptomatic women presenting with VP between 34 and 36 weeks' gestation.

- $\quad$ Although there is insufficient evidence to support the universal midgestation ultrasound screening for VP, recent evidence supporting the need for standardized prenatal targeted screening protocols of pregnancies at risk of VP. 


\section{REFERENCES AND RECOMMENDED READING}

Papers of particular interest, published within the annual period of review, have been highlighted as:

* of special interest

** of outstanding interest

1. Benirschke K, Burton GJ, Baergen RN. Pathology of the human placenta. $6^{\text {th }}$ edition. Berlin: Springer-Verlag; 2012.

2. Vahanian SA, Lavery JA, Ananth CV, Vintzileos A. Placental implantation abnormalities and risk of preterm delivery: a systematic review and metaanalysis. Am J Obstet Gynecol 2015; 213:S78-90.

3. Silver BM. Abnormal placentation: Placenta previa, vasa previa and placenta accreta. Obstet Gynecol 2015; 126:654-668.

4. Society of Maternal-Fetal (SMFM) Publications Committee, Sinkey RG, Odibo AO, Dashe JS. \#37: Diagnosis and management of vasa previa. Am J Obstet Gynecol 2015; 213:615-619.

5. Gagnon R, Morin L, Bly S, et al; Diagnostic Imaging Committee; Maternal Fetal Medicine Committee. SOGC clinical practice guidelines for the management of vasa previa. Int J Gynaecol Obstet 2010; 108:85-89.

6. Oyelese $\mathrm{Y}$, Catanzarite V, Prefumo F, et al. Vasa previa: the impact of prenatal diagnosis on outcomes. Obstet Gynecol 2004; 103:937-942.

7. Smorgick N, Tovbin $\mathrm{Y}$, Ushakov $\mathrm{F}$, et al. Is neonatal risk from vasa previa preventable? The 20 -year experience from a single medical center. $\mathrm{J}$ Clin Ultrasound 2010; 38:118-122. 
8. Vintzileos AM, Ananth CV, Smulian JC. Using ultrasound in the clinical management of placental implantation abnormalities. Am J Obstet Gynecol 2015; 213:S70-77.

9. Swank ML, Garite TJ, Maurel K, et al; Obstetrix Collaborative Research Network. Vasa previa: diagnosis and management. Am J Obstet Gynecol 2016; 215:223.e1-6. *

This large multicenter study confirms most current recommendations that include risk-based ultrasound screening, early hospitalization at 30-34 weeks gestation, antenatal corticosteroids at 30-32 weeks gestation, and elective delivery at 33-34 weeks gestation in pregnancies diagnosed with VP.

10. Melcer Y, Jauniaux E, Maymon S, et al. Impact of targeted scanning protocols on perinatal outcomes in pregnancies at risk of placenta accreta spectrum or vasa previa. Am J Obstet Gynecol 2018; 218:443.e1-443.e8.**

The first study to demonstrate that the implementation of prenatal standardised targeted screening protocols for placenta accreta spectrum and vasa previa in pregnant women at risk improves both maternal and neonatal obstetric outcomes.

11. Wood PL. Vasa paevia and placenta praevia screening in pregnancy. External review against programme appraisal criteria for the UK National Screening Committee, 2013

[legacyscreening.phe.org.uk/policydb_download.php]

12. Cipriano LE, Barth WH Jr, Zaric GS. The cost-effectiveness of targeted 
or universal screening for vasa praevia at 18-20 weeks of gestation in Ontario. BJOG 2010; 117:1108-1118.

13. UK National Screening Committee. Screening for vasa praevia in the second trimester of pregnancy. External review against programme appraisal criteria for the UK National Screening Committee (UK NSC). London: UK NSC; 2017 [https://legacyscreening.phe.org.uk/vasapraevia].

14. Räisänen S, Georgiadis L, Harju M, et al. Risk factors and adverse pregnancy outcomes among births affected by velamentous umbilical cord insertion: a retrospective population-based register study. Eur $\mathrm{J}$ Obstet Gynecol Reprod Biol 2012; 165:231-234.

15. Ebbing C, Kiserud T, Johnsen SL, et al. Prevalence, risk factors and outcomes of velamentous and marginal cord insertions: a populationbased study of 634,741 pregnancies. PLoS One 2013; 8:e70380.

16. Jauniaux E, Ben-Ami I, Maymon R. Do assisted-reproduction twin pregnancies require additional antenatal care? Reprod Biomed Online 2013; 26:107-119.

17. Hubinont $\mathrm{C}$, Lewi $\mathrm{L}$, Bernard $\mathrm{P}$, et al. Anomalies of the placenta and umbilical cord in twin gestations. Am J Obstet Gynecol 2015; 213:S91102.

18. Ruiter L, Kok N, Limpens J, et al. Incidence of and risk indicators for vasa praevia: a systematic review. BJOG 2016; 123:1278-1287. **

The first systematic review of 13 studies (two prospective cohort studies, 10 retrospective cohort studies and one case-control study), reporting on 569410 
women found a mean incidence of VP of 0.60 per 100 pregnancies and showing that $83 \%$ of the 325 VP cases reviewed had one or more risk factor.

19. Sullivan EA, Javid N, Duncombe G, et al. Vasa previa diagnosis, clinical practice, and outcomes in Australia. Obstet Gynecol 2017; 130:591$598 .^{* *}$

This large prospective study was performed using the Australasian Maternity Outcomes Surveillance System and shows that outcomes for neonates in which vasa previa was not diagnosed prenatally were inferior with higher rates of perinatal morbidity and mortality.

20. Jauniaux E, Melcer Y, Maymon R. Prenatal diagnosis and management of vasa previa in twin pregnancies: a case series and systematic review. Am J Obstet Gynecol 2017;216:568-575. **

The first systematic review on diagnosis and management of VP in twin pregnancies showing that management strategies in twin pregnancies presenting with vasa praevia should be tailored on the basis of chorionicity and changes in cervical length.

21. Jauniaux E, Englert $Y$, Vanesse M, et al. Pathologic features of placentas from singleton pregnancies obtained by in vitro fertilization and embryo transfer. Obstet Gynecol 1990; 76:61-64.

22. Schachter M, Tovbin $\mathrm{Y}$, Arieli S, et al. In vitro fertilization is a risk factor for vasa previa. Fertil Steril 2002; 78:642-643.

23. Baulies S, Maiz N, Muñoz A, et al. Prenatal ultrasound diagnosis of vasa praevia and analysis of risk factors. Prenat Diagn 2007; 27:595-599. 
24. Gavriil P, Jauniaux E, Leroy F. Pathologic examination of placentas from singleton and twin pregnancies obtained after in vitro fertilization and embryo transfer Pediatr Pathol 1993; 13:453-462.

25. Delbaere I, Goetgeluk S, Derom C, et al. Umbilical cord anomalies are more frequent in twins after assisted reproduction. Hum Reprod 2007; 22:2763-2767.

26. Suzuki S, Igarashi M, Inde Y, Miyake H. Abnormally shaped placentae in twin pregnancy. Arch Gynecol Obstet 2010; 281:65-69.

27. Gianopoulos J, Carver T, Tomich PG, et al. Diagnosis of vasa previa with ultrasonography. Obstet Gynecol 1987;69:488-491.

28. Hayata E, Nakata M, Oji A, et al. Four-dimensional spatiotemporal image correlation (4D-STIC) sonographic diagnosis of vasa previa. Ultrasound Obstet Gynecol 2018 May 28. doi: 10.1002/uog.19100. [Epub ahead of print]

29. Ruiter L, Kok N, Limpens J, et al. Systematic review of accuracy of ultrasound in the diagnosis of vasa previa. Ultrasound Obstet Gynecol. $2015 ; 45: 516-522$.

30. Nomiyama M, Toyota $\mathrm{Y}$, Kawano $\mathrm{H}$. Antenatal diagnosis of velamentous umbilical cord insertion and vasa previa with colour Doppler imaging. Ultrasound Obstet Gynecol 1998; 12:426-429.

31. Catanzarite V, Maida C, Thomas W, et al. Prenatal sonographic diagnosis of vasa previa: ultrasound findings and obstetric outcome in ten cases. Ultrasound Obstet Gynecol 2001; 18:109-115. 
32. Hasegawa J, Nakamura M, Sekizawa A, et al. Prediction of risk for vasa previa at 9-13 weeks' gestation. J Obstet Gynaecol Res 2011; 37:13461351.

33. Sinkey RG, Odibo AO. Vasa previa screening strategies: a decision and cost-effectiveness analysis. Ultrasound Obstet Gynecol 2018 May 22. doi: 10.1002/uog.19098. [Epub ahead of print] **

This study presents a decision and cost-effectiveness analysis comparing four screening strategies for the prenatal screening of VP in singleton pregnancies showing that screening pregnancies conceived by IVF is the most cost-effective strategy with an incremental cost effectiveness ratio of $\$ 29,186.50$ / quality adjusted life years.

34. Society of Maternal-Fetal (SMFM) Publications Committee, Sinkey RG, Odibo AO, Dashe JS. \#37: Diagnosis and management of vasa previa. Am J Obstet Gynecol 2015; 213:615-619.

35. Silver BM. Abnormal placentation: Placenta previa, vasa previa and placenta accreta. Obstet Gynecol 2015; 126:654-668.

36. Catanzarite V, Cousins L, Daneshmand S, et al. Prenatally diagnosed vasa previa: a single-institution series of 96 cases. Obstet Gynecol 2016; 128:1153-1561.*

A large single single-institution series of 96 women with prenatally diagnosed vasa previa showed that planned preterm delivery resulted in elective delivery for singletons in $62 \%$ and for twins $32 \%$. Gestational age at birth on average was 34.7 weeks for singletons and 32.8 weeks of gestation for twins. As a result 
of the high rate of emergent delivery in twins, delivery at 32-34 weeks of gestation may be risk-beneficial. The high rate of singletons without risk factors for vasa previa supports the recommendation to screen routinely for cord insertion site in high-risk patients.

37. Gagnon R. No. 231-Guidelines for the management of vasa previa. J Obstet Gynaecol Can 2017; 39:e415-e421.

38. Robinson BK, Grobman WA. Effectiveness of timing strategies for delivery of individuals with vasa previa. Obstet Gynecol 2011; 117:542549.

39. Moroz LA, Siimhan HN. Rate of sonographic cervical shortening and biologic pathways of spontaneous preterm birth. Am J Obstet Gynecol 2014; 210:555.e1-555.e5.

40. Ghi T, Contro E, Martina T, et al. Cervical length and risk of antepartum bleeding in women with complete placenta previa. Ultrasound Obstet Gynecol 2009; 33:209-212.

41. Zaitoun MM, El Behery MM, Abd El Hameed AA, Soliman BS. Does cervical length and the lower placental edge thickness measurement correlate with clinical outcome in cases of complete placenta previa? Arch Gynecol Obstet 2011; 284:867-873.

42. Maymon R, Melcer $\mathrm{Y}$, Tovbin J, et al. The rate of cervical length shortening in the management of vasa previa. J Ultrasound Med 2018; 37:717-723.*

This case-controlled study has found that the rate of CL shortening is significantly slower for women with elective caesarean delivery compared with 
those needing an emergency caesarean section. For each additional millimetreper-week decrease in CL, the odds of emergency caesarean delivery were increased by 6.50 (95\% Cl 1.02-41.20).

43. Melamed N, Pittini A, Hiersch L, et al. Do serial measurements of cervical length improve the prediction of preterm birth in asymptomatic women with twin gestations? Am J Obstet Gynecol 2016; 215:616.e1616.e14. 


\section{FIGURE LEGENDS}

Figure 1. Composite pictures (A-D) Detection of vasa previa (arrow) by transvaginal sonogram and color Doppler crossing the internal cervical os; (E-F) VP vessels (arrow) after delivery of the placenta.

Figure 2. Algorithm for diagnosis and management of VP in singleton pregnancies from the routine mid-gestation detailed anatomy scan developed using the data from the recent literature review.

Figure 3. Transabdominal ultrasound images at 20 weeks in a patient presenting with a low-lying placenta showing: A) a velamentous cord insertion (arrow) on grey-scale imaging and B) lateral VPs on colour Doppler imaging. 